\title{
Small-Pitch HgCdTe Photodetectors
}

\author{
W.E. TENNANT, ${ }^{1,2,3}$ D.J. GULBRANSEN,${ }^{1}$ A. ROLL,${ }^{1}$ M. CARMODY,${ }^{1}$ \\ D. EDWALL, ${ }^{1}$ A. JULIUS, ${ }^{1}$ P. DREISKE ${ }^{1}$ A. CHEN, ${ }^{1}$ W. MCLEVIGE, ${ }^{1}$ \\ S. FREEMAN, ${ }^{1}$ D. LEE, ${ }^{1}$ D.E. COOPER, ${ }^{1}$ and E. PIQUETTE ${ }^{1}$ \\ 1.-Teledyne Imaging Sensors, 5212 Verdugo Way, Camarillo, CA 93012, USA. 2.-e-mail: \\ William.Tennant@teledyne.com. 3.—e-mail: wetennant@roadrunner.com
}

\begin{abstract}
If we can make wavelength-sized detectors, we approach the limit at which smaller detectors have no further advantage for imaging focal plane arrays with practical (f/1-2) optics. Of course, this must be accomplished without compromising performance-a challenge for 5- $\mu \mathrm{m}$ devices for which the perimeter, the currents of which depend on passivation quality, is very large compared with the area of the device. This paper describes the development of small LWIR HgCdTe detectors and compares dark current performance with that of larger basic devices, as described by "Rule 07", a well-known rule of thumb which gives the HgCdTe dark-current density characteristics of the best reported diodes as a function of device cut-off wavelength and operating temperature. Low cross-talk requires a fully-depleted absorber layer sufficiently thick to provide adequate quantum efficiency (QE). Preliminary results show dark-current densities are more than a factor of ten below the Rule 07 trend line. With these dark-current densities, the measured $\sim 40 \%$ non-antireflection-coated $\mathrm{QE}$ in the $8-10 \mu \mathrm{m}$ region is more than adequate to achieve background-limited performance with the margin under tactical backgrounds for the fast (f/1), diffraction-limited optics required for the small pixels.
\end{abstract}

Key words: Small pixel, lambda-scale, LWIR, HgCdTe, full depletion, rule 07, focal plane array (FPA), infrared, diffraction-limited, low dark current

\section{INTRODUCTION: BACKGROUND AND STATEMENT OF THE PROBLEM}

Dr Nibir Dhar of the Defense Advanced Research Projects Agency (DARPA) presented a keynote SPIE paper "Advanced Imaging Research and Development at DARPA"1 in which he discussed the Advanced Wide FOV Architecture for Image Reconstruction and Exploitation (AWARE)-Lambda Scale detector program, which is developing high-performance focal plane arrays (FPA) with pixel dimensions approaching the wavelength scale (Nyquist limit). In this program, unit cells are being reduced to $5 \mu \mathrm{m} \times 5 \mu \mathrm{m}$ for LWIR and MWIR. HgCdTe has been the detector material of choice for this activity. With pixel pitch approximately half the LWIR-detected wavelength, these arrays will be diffraction-limited in any optical

(Received September 28, 2013; accepted April 15, 2014; published online June 3, 2014) system slower than $\mathrm{f} / 1$. Such small-pitch detectors will be the focus of this paper. Other key technology developments, for example interconnect density and high charge capacity, will be discussed elsewhere.

The small pixel pitch requirement challenges both detector performance and processing. To achieve low cross-talk from lateral diffusion requires a fullydepleted absorber layer. Full absorber depletion must be obtained at sufficiently low bias to prevent tunneling, but with a layer sufficiently thick to provide adequate quantum efficiency $(\mathrm{QE})$. This combination of requirements necessitates low absorber doping and robust diode reverse-bias characteristics. Full absorber depletion also may suppress Auger recombination, in which case the ultimate dark current achieved will depend on both residual generation-recombination (GR) currents from the depletion region and on p-side and peripheral bulk and surface contributions. All these limiting contributions are most difficult to minimize for a small detector pixel. 
Our preliminary results show that such devices have dark-current densities more than a factor of ten below the Rule 07 trend line, ${ }^{2,3}$ because of their low doping and thin layer structure. With these darkcurrent densities, the measured $\sim 40 \%$ non-antireflection (non-AR)-coated $\mathrm{QE}$ in the $8-10 \mu \mathrm{m}$ region is more than adequate to achieve background-limited performance (BLIP) with the margin under tactical backgrounds $\sim 5 \times 10^{16} / \mathrm{cm}^{2}$-s for the fast (f/1), diffraction-limited optics required for the small pixels. To illustrate this, assume an AR-coated $Q E$ of $31.5 \%$ and effective $\mathrm{f} / 1$ optics transmission of $70 \%$, which results in a background flux at the detector of $4 \times 10^{16} / \mathrm{cm}^{2}-\mathrm{s}$ and a photocurrent density of $2.8 \mathrm{~mA} / \mathrm{cm}^{2}$. For comparison, a $78 \mathrm{~K} 11.5 \mu \mathrm{m}$ cut-off Rule 07 photodiode has a dark-current density of $<70 \mu \mathrm{A} / \mathrm{cm}^{2}$-approximately a factor of 40 lower than the photocurrent, and insignificant by comparison. The Rule-07-beating detectors reported in this paper are, therefore, BLIP by a factor of $\sim 100$. Simple calculation of the incident $300 \mathrm{~K}$ blackbody flux shows that integrating for less than $2.5 \mathrm{~ms}$ will give a noise equivalent difference in temperature (NEDT) below $30 \mathrm{mK}$ for a $5 \mu \mathrm{m}$-pitch photodiode.

\section{Approach to Improved Detectors}

To support the fully depleted device architecture required for high-resolution small pixels, we investigated a variety of different device geometries. The basic architecture used was Teledyne's double layer planar heterostructure (DLPH) described in detail elsewhere. ${ }^{4,5}$ Although device geometry varied, all involved reduction of impurity doping, thinning of active layers, reducing dislocations, and optimizing junction position. We attribute the low dark currents of these devices to the low doping and thinness of the layers coupled with the devices being limited by fundamental Auger-generated dark current. Tennant and others (Ref. 3 and references cited therein) suggested it might be possible to obtain dark-current densities well below those of Rule 07 by substantially reducing the free carriers in the semiconductor by full depletion. In this work reduction of free carriers was achieved by reducing doping of the absorber layer, and by some depletion. It is also well known that the dark-current density increases with absorber layer thickness, so thinning and reduction of the doping led to substantially lower dark currents.

Fabrication of these $5 \mu \mathrm{m}$-pitch devices poses several challenges, most of which can be overcome by high-quality photolithography. By use of a highresolution I-line stepper mask aligner we were able to use Teledyne's DLPH architecture for this small pitch.

\section{Evaluation of High-Performance Small-Pitch Devices}

During the program we grew several wafers, most of which resulted in excellent device performance.
Table I below shows process-evaluation chip (PEC) device performance for the ten best wafers. Some adjustment of wavelength and thickness was made for the later layers $(\mathrm{G}-\mathrm{H})$, because it was clear from the first lots that we did not need such a long wavelength to obtain high quantum efficiency in the 8-10 $\mu \mathrm{m}$ spectral region, even with thin layers. This slight increase in thickness and Cd fraction gave the same $8.1 \mu \mathrm{m}$ quantum efficiency, but with a shorter cut-off and a (sometimes) lower dark-current density. The dark-current density reported in the table is typically that of a $40 \times 40$ element mini-array of $5 \mu \mathrm{m}$-pitch detectors.

Because of the thinness of these layers, they are not well described by a "cut-off" wavelength. Figure 1 shows the average $80 \mathrm{~K}$ spectral quantum efficiency measured for several large diodes in the PEC from wafer E. Using the definition of the cut-off wavelength as the wavelength at which responsivity reaches half the peak value, the cut-off wavelength is $12.7 \mu \mathrm{m}$. In the $7.8-10.1 \mu \mathrm{m}$ spectral region quantum efficiency averages over $45 \%$ in the absence of AR coating. A one-dimensional model (assuming a reflective contact) was fit to the data points by varying only thickness and cadmium mole fraction $(x)$; this also appears in the figure. This model is a derived from the work of Williams and DeWames, ${ }^{6}$ but simplified and modified slightly. ${ }^{7}$ The resulting good-quality fit curve (also shown in the figure) gives a Cd mole fraction ( $x$ ) of 0.207 and a thickness of $1.17 \mu \mathrm{m}$. It is likely that the actual thickness is slightly greater and the layer $\mathrm{Cd}$ mole fraction is somewhat graded, with the model giving a thinner, slightly longer estimate for an ungraded compositional profile. It is important to note that, despite the graded response, the quantum efficiency in the $8-10 \mu \mathrm{m}$ spectral range is more than sufficient to enable high charge collection in a relatively short $(<3 \mathrm{~ms})$ integration time, as was mentioned above, because high $\mathrm{HgCdTe}$ quality results in low dark currents, even for extended wavelengths, and in an abundance of flux in the LWIR spectral region. Having a thin layer which can be fully depleted to reduce cross-talk is a worthwhile exchange for a modest decrease in quantum efficiency.

The same model used to fit the data in Fig. 1 was used to obtain an estimate of the expected $78 \mathrm{~K}$ dark-current density, assuming nominal doping of $3 \times 10^{14} / \mathrm{cm}^{3}$ and Auger and radiative generation. The model gives a current density of $17 \mu \mathrm{A} / \mathrm{cm}^{2}$ whereas measured values were approximately $40 \mu \mathrm{A} / \mathrm{cm}^{2}\left(1 \times 10^{-11} \mathrm{~A}\right)$ for $1 \times 15 \mu \mathrm{m}$ devices and $53 \mu \mathrm{A} / \mathrm{cm}^{2}\left(2.1 \times 10^{-8} \mathrm{~A}\right)$ for $40 \times 405 \mu \mathrm{m}$ devices. This is apparent from Fig. 2, which shows the current-voltage $(I-V)$ characteristics both for $40 \times 40$ mini-arrays of diodes (with a common contact making them act as a single diode) and for singleelement devices. It should be noted that current and current density are taken from the flat reversebiased saturation region (in the vicinity of -100 to $-200 \mathrm{mV}$ bias), because this is the natural 
Table I. PEC device performance data for LWIR HgCdTe $5 \mu$ m-pitch detectors compared with the Rule 07 trend line

\begin{tabular}{|c|c|c|c|c|}
\hline Wafer & $\begin{array}{c}\mathrm{QE} \\
(\% \text { at } 8.1 \mu \mathrm{m} \text { and } \\
80 \mathrm{~K}, \text { not } \mathrm{AR} \text {-coated })\end{array}$ & $\begin{array}{c}50 \% \text { response } \\
\text { cut-off wavelength } \\
(\mu \mathrm{m})\end{array}$ & $\begin{array}{c}\text { Dark-current } \\
\text { density }\left(\mu \mathrm{A} / \mathrm{cm}^{2} \text { at } 78 \mathrm{~K}\right)\end{array}$ & $\begin{array}{c}\text { Rule 07 trend } \\
\text { line/measured } \\
\text { dark-current density }\end{array}$ \\
\hline $\mathrm{A}$ & 50 & 12.1 & 14 & 11.9 \\
\hline B & 53 & 12.4 & 51 & 5.0 \\
\hline $\mathrm{C}$ & 45 & 13.5 & 161 & 6.5 \\
\hline $\mathrm{D}$ & 67 & 12.6 & 23 & 14.6 \\
\hline $\mathrm{E}$ & 52 & 12.6 & 40 & 8.4 \\
\hline $\mathrm{F}$ & 43 & 12.7 & 123 & 3.1 \\
\hline $\mathrm{G}$ & 55 & 10.7 & 4.3 & 3.7 \\
\hline $\mathrm{H}$ & 53 & 10.7 & 17 & 0.9 \\
\hline I & 58 & 11.1 & 6 & 5.7 \\
\hline$J$ & 58 & 11.3 & 98 & 0.5 \\
\hline
\end{tabular}

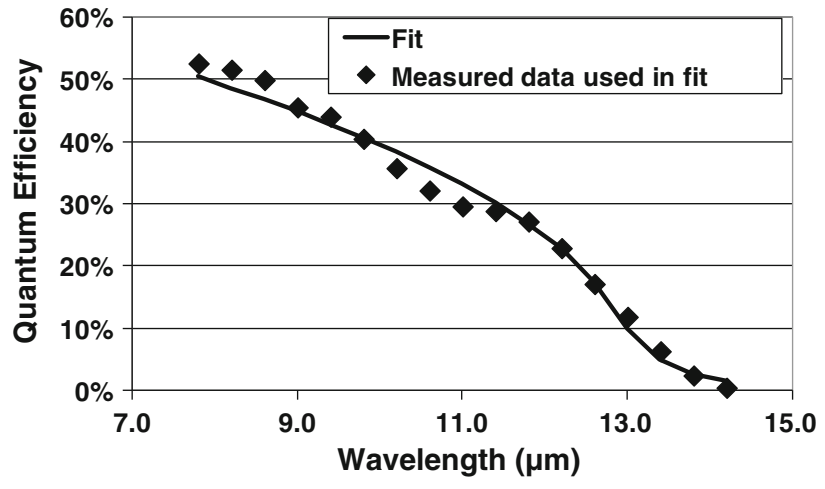

Fig. 1. Average spectral quantum efficiency of several diodes in the PEC from wafer E. The model used to fit the data (use of the leastsquares difference normalized to the estimated measurement error) indicated a good quality of fit.

operating region for detectors and is well described by the ideal diffusion-limited diode equation $J=J_{\mathrm{D}}$ $\left[\exp \left(V / V_{T}\right)-1\right]$, where $J_{\mathrm{D}}$ is the saturation current density, $V$ is the applied voltage, and $V_{\mathrm{T}}$ is the thermal voltage $(=k T / q$, where $k$ is Boltzmann's constant, $T$ is the absolute temperature, and $q$ is the electronic charge). $J_{\mathrm{D}}$ is the value calculated by use of our 1D model. Here $J_{\mathrm{D}}=q^{*} n_{\mathrm{i}}^{2} b / N_{\mathrm{D}} \tau$, where $n_{\mathrm{i}}$ is the intrinsic carrier concentration, $N_{\mathrm{D}}$ is the doping density, $b$ is the layer thickness, and $\tau$ is the minority carrier lifetime. If the devices are limited by Auger processes, $\tau$ is proportional to $1 / N_{\mathrm{D}}^{2}$. Values for these properties of the materials can be obtained from the equations in the model reported in Ref. 7.

Both types of device are surrounded by a single ring of "guard" diodes identical with the diodes under test. The guard diodes serve to draw off peripheral currents, as would be achieved in an array by the diodes neighboring any particular detector element. Note that using reversed-bias guards to eliminate peripheral currents enables a 1D model to provide a good estimate of reverse-bias

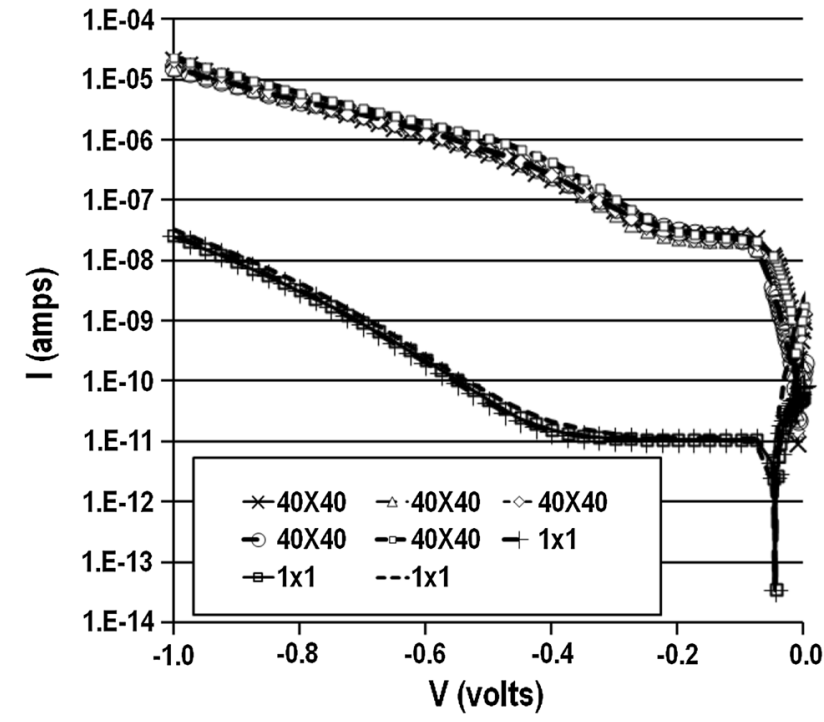

Fig. 2. Absolute value of the diode current as a function of reverse bias voltage for several "guarded" mini-arrays $(40 \times 40$ and $1 \times 1$, or single element) of 5 - $\mu \mathrm{m}$-pitch diodes from wafer $\mathrm{E}$. Dark-current densities are $40 \mu \mathrm{A} / \mathrm{cm}^{2}$ for the single elements and $53 \mu \mathrm{A} / \mathrm{cm}^{2}$ for the $40 \times 40$ mini-arrays.

device performance. Because of the presence of the guard diodes, however, the ideal equation above does not describe well the behavior near zero bias. The guard diodes in the figure were held at $-300 \mathrm{mV}$ bias, causing the slight shift of the zero current point to negative bias voltages (seen as the negative voltage values of the low-current "spikes" in the curves of Fig. 2). For reverse bias magnitudes greater than $350 \mathrm{mV}$ for the single elements and $250 \mathrm{mV}$ for the mini-arrays, tunneling currents begin to dominate.

These diode dark-current densities are a factor of 7-8 lower than our "Rule 07" trend line. ${ }^{6,7}$ However, this low value is still consistent with Auger 1 recombination. If the lower doping densities (a factor of 1.3-3.3) and the thinner layers (a factor of 
4-10) of these devices are taken into account, one might expect dark-current density reduction by a factor of 5-33 compared with the Auger performance of a typical "Rule 07" diode (with 0.4$1.0 \times 10^{15} / \mathrm{cm}^{3}$ doping and thickness equal to the cut-off wavelength). This range includes 6 of the 10 layers in Table I. It should also be noted that although the target doping level was $~ 3 \times 10^{14} / \mathrm{cm}^{3}$, actual doping of the layer may have varied somewhat from wafer to wafer, creating additional deviations from the results from our dark-current density model.

Figure 3 compares results obtained with a "prebiased" guard diode with those for the guard diode held at (approximately) the same bias as the diode under test. For measurement of these devices, we initially biased both the guard diode and the diode under test at the same potential. This led to a "negative resistance" region in reverse bias because of gradual "pinching-off" by the guard of large lateral currents, as mentioned above. This can be seen from the figure by comparing $J-V$ for the diode with the guard held at the diode potential with that for the diode with the guard pre-biased at $-300 \mathrm{mV}$. The solid curve is for the pre-biased guard diode and shows the abovementioned negative shift in voltage corresponding to zero current. Comparison of the dashed curve at $\sim-50 \mathrm{mV}$ with the solid curve at approximately the same bias (just after the solid curve current becomes level), and noting that the dashed curve lies close to the solid curve at higher biases, reveals that the peripheral dark current contribution is reduced or "pinched off" (by approximately a factor of 100) because of the bias on the single $5 \mu \mathrm{m}$-wide guard diode. If the two diodes (that under test and the guard) were at exactly the same bias, we would expect the currents to coincide at $-300 \mathrm{mV}$. That they do not coincide is probably indicative of a slight difference in the bias potential because of small measurement apparatus bias offsets between the diode under test and the guard. This pinching off of the lateral currents indicates that we have fully depleted the layer at a modest bias of approximately $100 \mathrm{mV}$, and further indicates cross talk between pixels should be very low. The $100 \mathrm{mV}$ bias is consistent with a depletion width of $\sim 1 \mu \mathrm{m}$, which is comparable with the effective layer thickness.

\section{FPA Evaluation of $5 \boldsymbol{\mu m}$-Pitch Diodes}

We evaluated these $5 \mu \mathrm{m}$ pixels in a hybrid FPA configuration by combining pixels into groups (typically $3 \times 3$ pixels) with a common contact and connecting each of these $15 \mu \mathrm{m}$-pitch groups to the input of a $15 \mu \mathrm{m}$-pitch $640 \times 512$ direct injection readout integrated circuit (ROIC). Figure 4 illustrates this concept.

Figure 5 shows the spectral quantum efficiency of a $250-\mu \mathrm{m}$ diode in the PEC from the wafer used for this hybrid FPA device. In the absence of AR

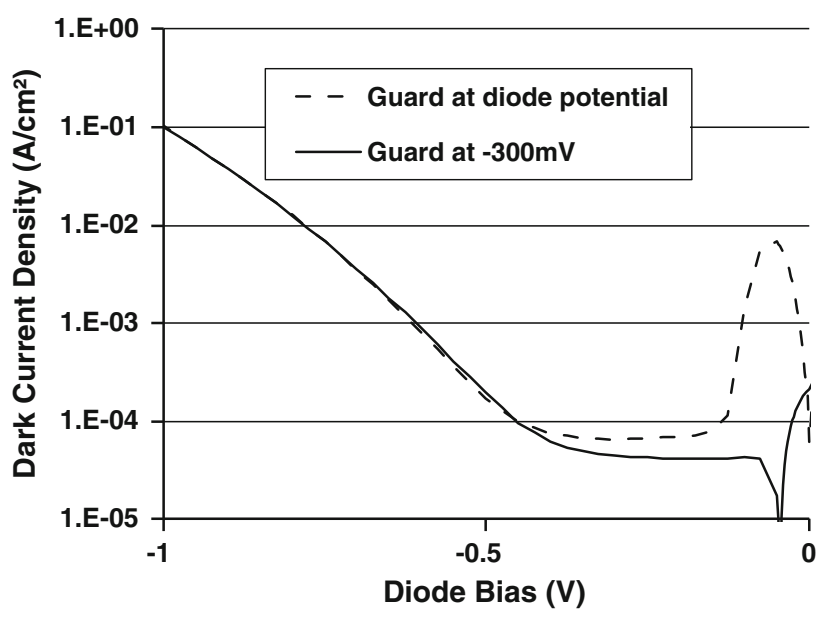

Fig. 3. Absolute value of dark-current density as a function of reverse bias voltage for a guarded $1 \times 1$ element $(5 \mu \mathrm{m})$ diode with the guard pre-biased to $-300 \mathrm{mV}$ compared with the guard held at the same bias as the tested diode.

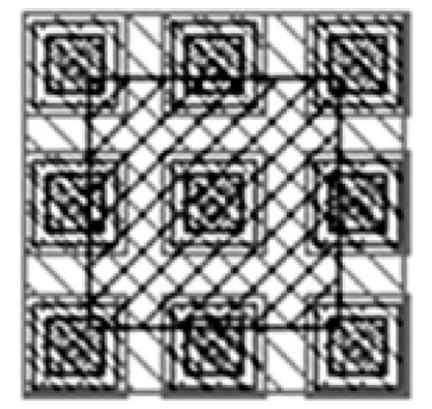

Fig. 4. Nine-5 $\mu$ m-pitch detectors connected through a common metal-level contact pad to a single $15 \mu \mathrm{m}$-pitch ROIC pixel.

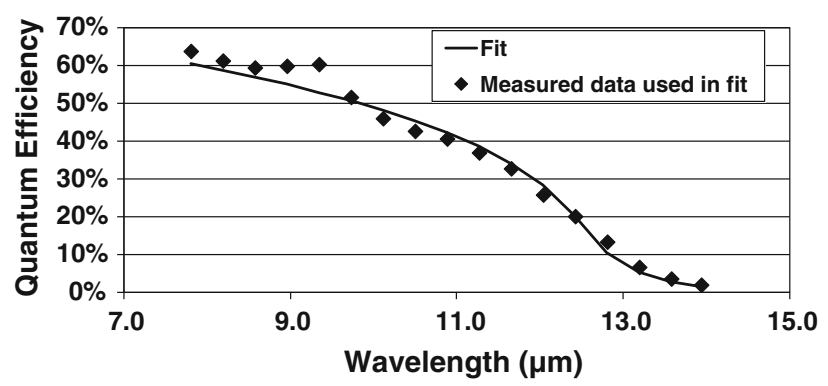

Fig. 5. Spectral quantum efficiency data from a process-evaluation chip (PEC) from the same wafer B used to make the banded FPA. A 1D non-graded model fit to the curve adjusting only for thickness and $\mathrm{Cd}$ mole fraction, and assuming a reflective contact, gave the highquality fit shown.

coating, in the 7.8-10.1- $\mu \mathrm{m}$ spectral region average quantum efficiency is $>57 \%$. As described above, a one-dimensional model (assuming a reflective contact) was fit to the data points by varying only thickness and cadmium mole fraction. The resulting good-quality fit curve (also shown in the figure) gave a cadmium mole fraction, $x$, of 0.2087 and a 

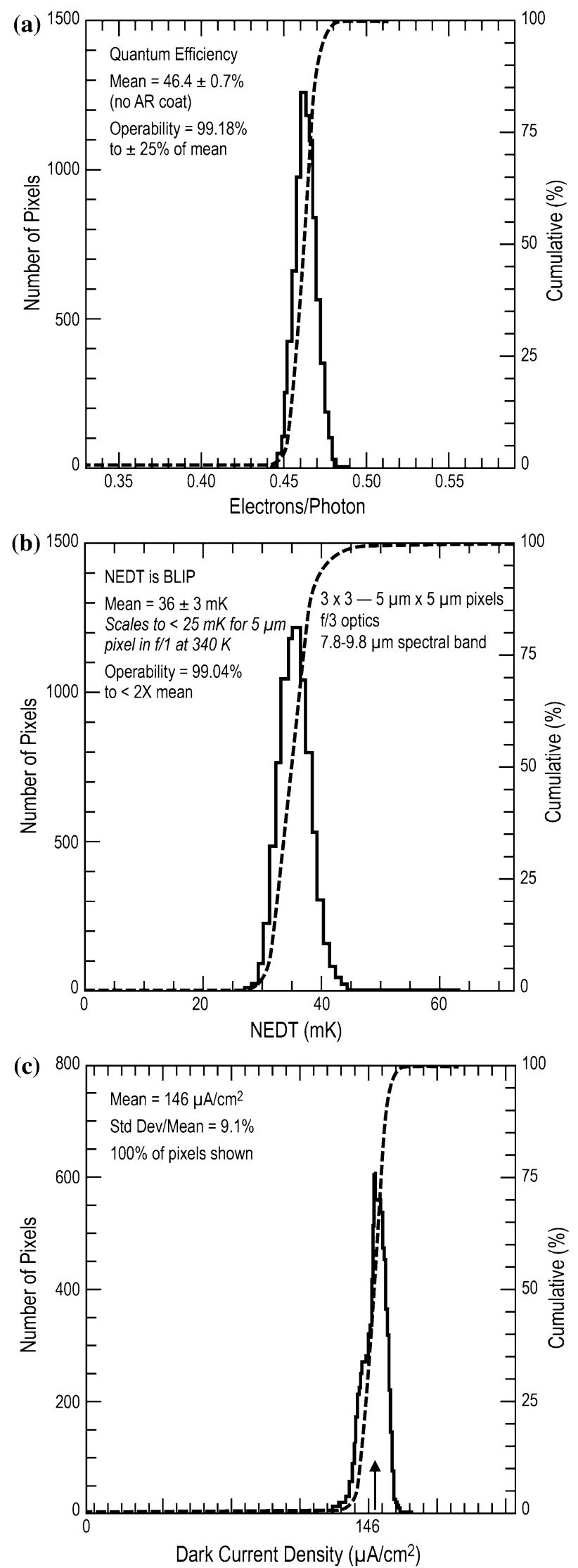

Fig. 6. Histograms of quantum efficiency (a), NEDT (b), and darkcurrent density (c) for an FPA using a $15 \mu \mathrm{m}$-pitch ROIC to connect to $3 \times 3$ arrays of $5 \mu \mathrm{m}$-pitch diodes. thickness of $1.74 \mu \mathrm{m}$. In this case it is likely that the actual thickness is slightly greater and the layer $\mathrm{Cd}$ mole fraction is somewhat graded, as for the previous layer discussed above, with the model giving a thinner, slightly longer estimate for an ungraded compositional profile. The spectral response cut-off is $12.42 \mu \mathrm{m}$.

This was a banded FPA with different regions having different architecture (e.g. varying implant size, contact size, etc.) or grouping. Several different types of architecture were used, each implemented in bands of 16 columns by 512 rows of ROIC inputs $(8,192$ pixels or $73,7285-\mu \mathrm{m}$ detectors per band). Each band was repeated two or three times. Quantum efficiency, noise equivalent difference in temperature (NEDT), and dark-current density of the best band are shown in Fig. 6 . All the values show performance, uniformity, and operability were excellent. Quantum efficiency of $46 \pm 0.7 \%$ (in the spectral range from 7.8 to $9.8 \mu \mathrm{m}$ ) for a non-ARcoated part is equivalent to $>55 \%$ with an $\mathrm{AR}$ coating; and its outstanding uniformity (1.5\% sigma/ mean) is as good as we have obtained in any $\mathrm{HgCdTe}$ FPA measurement. This uniformity resulted in operability of $>99 \%$ in the range $\pm 25 \%$ of the mean. Note that the higher quantum efficiency observed for the PEC in this spectral region may, at least in part, be because the PEC device had a fully reflecting contact whereas the FPA had regions between the contact pads where the surface was probably not fully reflective.

The mean value of the NEDT is $36 \pm 3 \mathrm{mK}$ (for a $300 \mathrm{~K}$ scene temperature) with operability over $99 \%$ in the range of twice the mean, and was approximately BLIP-limited, with the primary constraint being the limited charge capacity of the readout (designed for a different purpose). With a contrast ratio of 0.0182 for this spectral region, a $36-\mathrm{mK}$ BLIP NEDT would require integration for approximately 2.3 million carriers; however, some dark current and ROIC read noise contribution required integration for somewhat more carriers. The $8 \%$ spread of the NEDT histogram (which is quite uniform and symmetrical) is likely to arise from the statistics of $\sim 100$ sample frames. The dark-current density of 146 $\mu \mathrm{A} / \mathrm{cm}^{2}$ is approximately $60 \%$ of that which one would expect from the Teledyne "Rule 07" trend line. This is not surprising, considering the low doping and thinness of the active layer, although it is approximately $3 \times$ higher than the dark-current density of the miniarrays reported in Table I. Because the high uniformity of the dark current suggests the diode currents are limited by properties of the uniform material rather than any process-induced defect, we speculate that this difference from PEC measurements may be because the FPA was not quite as cold as the temperature sensor indicated. Although the FPA operating temperature was nominally $78 \mathrm{~K}$, operation at $83 \mathrm{~K}$ would fully account for the difference between PEC and FPA dark current measurement. Some of the detector dark currents observed may also be 
generated from nearby surfaces and interfaces (which differ slightly between FPA and PEC mini-arrays because of the extent of metallization overcoating). If so, further dark current reduction and quantum efficiency improvement may be possible by further refinement of growth and passivation and by increasing the fractional coverage of the contact metal.

\section{CONCLUSIONS}

We report the first planar $5 \mu \mathrm{m}$-pitch detectors made by ion implantation in molecular beam epitaxy-grown $\mathrm{HgCdTe}$ heterostructures. Detector architecture is a modification of Teledyne's DLPH. The performance of these devices often substantially exceeds that of the $\mathrm{HgCdTe}$ trend line as epitomized by Teledyne's Rule 07. This low dark current is explained by the lower doping and active layer thickness of these wafers, with the associated reduction in Auger-limited diffusion dark currents, compared with the wafers used to develop Rule 07. Analysis of an FPA of combined small (nineelement) mini-arrays shows these small-pitch devices have potential for high performance and operability. This paper provides fundamental insights and evidence that proper attention to doping and layer thickness in an Auger-limited detector material, for example $\mathrm{HgCdTe}$, can lead to a substantially reduced dark current while maintaining high sensitivity. Good device design can preserve this fundamental performance and achieve low cross-talk, even for detectors as small as 5-micron pitch. Thus, while still a good rule of thumb for standard $\mathrm{HgCdTe}$ detectors, Rule 07 can be surpassed by use of device architecture based on appropriate physical understanding.

\section{ACKNOWLEDGEMENTS}

Work supported by the Defense Advanced Research Projects Agency, contract number W31P4Q-09-C0513, Dr. Nibir Dhar, Program Manager.

\section{OPEN ACCESS}

This article is distributed under the terms of the Creative Commons Attribution License which permits any use, distribution, and reproduction in any medium, provided the original author(s) and the source are credited.

\section{REFERENCES}

1. N.K. Dhar and R. Dat, Proc. SPIE 8353, 835302 (2012).

2. W.E. Tennant, D. Lee, M. Zandian, E. Piquette, and M. Carmody, J. Electron. Mater. 37, 1406 (2008).

3. W.E. Tennant, J. Electron. Mater. 39, 1030 (2010).

4. J. Bajaj, J.M. Arias, M. Zandian, D.D. Edwall, J.G. Pasko, L.O. Bubulac, and L.J. Kozlowski, J. Electron. Mater. 25, 1394 (1996).

5. J.M. Arias, J.G. Pasko, M. Zandian, J. Bajaj, L.J. Kozlowski, R.E. DeWames, and W.E. Tennant, Proc. SPIE 2228, 210 (1994).

6. G.M. Williams and R.E. DeWames, J. Electron. Mater. 24, 1239 (1995).

7. W.E. Tennant, J. Prog. Quantum Electron. 36, 273 (2012). 